\title{
12. Experimental Study on Brain Hemorrhage
}

\author{
Junichi Wakisaka, Shinken Kuramoto, Sigeyuki TakaI \\ and Shigeyuki BABA \\ 1st Surgical Department, Kurume University School of Medicine
}

An evaluation was made on cerebrospinal fluid pressure, rectal temperature, leucoyte, blood sugar, cerebrospinal fluid and serum transaminase which occurred in the brain hemorrhage of animals, purposely prepared for clinical experiment. Matured dogs, weighting approximately $10 \mathrm{~kg}$ were utilized, and the Tokyo Uniuersity Brain Laboratory Type Animal Stabilizer was adopted for this experiment, and self made arterial blood was injected at the rate of $1 \mathrm{cc}$ per kilogram of the dogs weight into the intraventricular and the internal capsular.

The cerebrospinal fluid pressure rose 10 to 12 fold, simultaneous to the injection of the arterial blood, but the drop of the pressure continued for 2 hours thereafter, and began rising again 3 hours later. Approximately the same symptom was noticed in the blood pressure. The rectal temperature began rising rapidly after the 3 rd hour, and the same action was seen in the leucocyte. The increase in the amount of blood sugar continued until the 4 th hour, and in most cases a great amount of blood sugar was noticed after the 4 th hour.

In regard to serum transaminase, GOT indicated a significant rise after the 12 th hour. The mortality in the intraventricular bleeding group showed $11.7 \%$ during the $3 \mathrm{rd}$ through the 12 th hour period, and $58.8 \%$ during the 12 th through the 48 th hour period, and the rates in the internal capsular bleeding group were $7.7 \%$ and $53.8 \%$, respectively.

The above mentioned facts indicate that in regard to experimentally prepared severe brain hemorrhage, the first 3 hours, after the bleeding operation being performed, is considered to be the confused period, in regard to the stress or the bleeding. During this period the fluid pressure rise rapidly and then drops, and the amount of blood sugar greatly increases, and significant changes are recognized in the blood pressure, in the rectal temperature and in the leucocyte. During the period between the 3rd through the 12 th hour, a conspicuous rise in the rectal temperature and a great increase in the amount of lecucocyte are noticed, and also during this period the cerebrospinal fluid pressure begins to rise again. This period is considered to be the reaction period which the bios reaction shows a set tendency. Further, during the 12 th through the 48 th hour period, cerebrospinal fluid GOT indicates a significant rise; and in both groups, the intraventricular and 
internal capsular bleeding groups, the rate of death is noticed to be over $50 \%$. This period may be called the critical period.

\title{
13. Experimental Study on Surgical Treatment of Hypertensive Intracerebral Hemorrhage (Second Report)
}

\author{
Osamu Mōri, Akira Nishimoto, Shuzō Okumura, Takurō Ogata, \\ Takao Kawashima, Kōzi Satō and Kazuhiko Sadamoto \\ Dept. of Neurological Surgery, Okayama University Medical School
}

Intracerebral hematoma was experimentally produced by injection of arterial autoblood into the area of the internal capsule with stereotaximetry in dogs. After 48 hours the hematoma was aspirated, and the aspirated and the non-aspirated cases were followed electro-encephalographically. The animals were sacrificed after one week to two months, whose brains were histologically and histochemically studied.

The activity of malic-, $\alpha$-glycerophosphate-, and glutamic acid-dehydrogenase in nerve cells was markedly decreased in areas surrounding the hematoma even after two months in non-aspirated cases, while it was well preserved in aspirated cases.

EEG recording showed localized or generalized slow wave activity immediately after the intracerebral blood injection, which continued even after two months in non-aspirated cases. Marked decrease of the slow activity was observed shortly after aspiration and EEG returned to almost normal pattern after one week.

Two patients of hypertensive intracerebral hemorrhage were operated and hematomas were surgically removed. One patient with hematoma of $100 \mathrm{~m} l$ in the left temporal lobe recovered very well, and six months later, he regained almost full activity except for slight motor aphasia. The other died on the first postoperative day. Angiographic and ultrasonic examination played most important role for localization of the site of hematoma in both cases. 